\title{
Article \\ Characterization of Recombinant Poliovirus 2A Protease; A Potential Anti-Viral Drug Target
}

\author{
Amna Younus ${ }^{1}$, Muhammad Faraz Bhatti ${ }^{1 *}$, Nasar Virk ${ }^{1}$, Muhammad Arshad ${ }^{2}$, Hussnain Ahmed \\ Janjua ${ }^{1}$, Robert H. A. Coutts ${ }^{3}$ \\ 1 Atta-ur-Rahman School of Applied Biosciences (ASAB), National University of Sciences and Technology \\ (NUST), Sector H-12, Kashmir Highway, Islamabad 44000, Pakistan; amna.11.dirphd.07@asab.nust.edu.pk; \\ mfbhatti@asab.nust.edu.pk; nasarvirk@asab.nust.edu.pk; hussnain.janjua@asab.nust.edu.pk \\ 2 Department of Bioinformatics and Biotechnology, International Islamic University, Sector H-10, Islamabad \\ 44000, Pakistan; m.arshad@iiu.edu.pk \\ 3 Department of Biological and Environmental Sciences, School of Life and Medical Sciences, University of \\ Hertfordshire, United Kingdom; r.coutts@herts.ac.uk \\ * Correspondence: mfbhatti@asab.nust.edu.pk; Tel.: 0092-51-90856136; Fax: 0092-51-90856102
}

\begin{abstract}
Poliovirus 2A protease (PV2Apro) plays a vital role in viral replication and downregulation of host cell protein synthesis. In order to understand more concerning PV2Apro, the protein was over-expressed in bacteria following amplification using sense and antisense primers and cloning in pET15b. Several expression hosts were tested and BL21 (DE3) pLysS cells gave the best expression of PV2A pro with minimal unwanted protein expression following IPTG induction. The $2 \mathrm{~A}^{\text {pro }}$ protein was purified to homogeneity using column chromatography, its solubility determined and its molecular weight and composition determined by MALDI-TOF mass spectrometry. The protease was found in the insoluble fraction and the purified protein had a slightly lower molecular weight than predicted. Moreover, three dimensional structure was modelled using template $1 \mathrm{z} 8 \mathrm{r}$ with $58 \%$ identity and validated using ramachandran plot. Results revealed that most of the residues lie in favoured and allowed regions. These findings could help in a better understanding of PV2A pro structure and inhibition thus, highlighting potential targets for antiviral drug development.
\end{abstract}

Keywords: poliovirus 2A protease (PV2A pro); expression; purification; MALDI-TOF; 3D structure

\section{Introduction}

Poliovirus (PV), causes poliomyelitis and is a major financial problem to developing countries trying to eradicate the disease and Pakistan is top of this list. For instance live PV vaccine strains have produced unanticipated hurdles, posing new serious obstacles for the "end game" in the eradication campaign [1].

The PV the prototypic picornavirus of family Picornaviridae comprises a single-stranded, positive-sense RNA ca. 7440 nucleotides long and serves as a messenger for translation [2]. During infection, the viral RNA is transferred into the host cell cytoplasm and translated into a single autocatalytic polyprotein $c a .250 \mathrm{kDa}$ in size [3]. This polyprotein is cleaved as a nascent chain into primary precursors which are further processed proteolytically producing structural and nonstructural proteins [4-7]. The PV genome encodes 3Cpro, 2Apro, and 3CDpro, all of which are involved in polyprotein processing. In the polyproteins, glutamine-glycine sites are cleaved by $3 \mathrm{C}$ pro and $3 \mathrm{CD}$ pro, while $2 \mathrm{~A}$ pro cleavage involves only tyrosine-glycine bonds [8]. The $2 \mathrm{~A}^{\text {pro }}$ and $3 \mathrm{C}$ pro proteins have a significant role in activating the apoptotic process in PV-infected cells and also exhibit an anti-apoptotic activity [1].

The $2 \mathrm{~A}$ protease belongs to a small cysteine-rich proteinase family and is similar to trypsin-like small serine proteases. To form a catalytic region in the active site of the $2 \mathrm{~A}$ protease a cysteine residue is substituted with a serine residue. There is no significant sequence similarity between 
cardio-, aphtho- or enteroviruses 2A proteins [9]. During replication a PV internal ribosome entry site (IRES) is stimulated after cleavage of the translational initiation factor eIF4G [10-12]. The stability of PV RNA involves 2Apro along with its prolonged translation in vivo, with no effect on RNA stabilization [13] enhancing viral protein synthesis. Furthermore, it has been demonstrated in PV infected HeLa cells that PV2A pro cleaves nuclear pore proteins concerned with envelope permeability [14-16]. Little is known concerning the involvement of 2A in PV RNA replication but it is suspected that it possibly contributes a role in the process [8].

Additionally, PV 2Apro is involved in host cell translational shutoff [17-18] mediated by the cleavage of p220, a component of the cap-binding initiation factor eIF-4F [19-20]. PV2Apro also prompts DNA fragmentation and chromatin condensation [21]. In the PV polyprotein translation process $2 \mathrm{~A}$ pro is thought to cleave the polyprotein in cis following refolding into an active conformation [22]. Moreover transient expression of $2 \mathrm{~A}^{\text {pro }}$ in eukaryotic cells inhibited both cellular translation and transcription [23]. Also, 2Apro induces cytopathic effects in infected cells and contributes to evading the inhibitory effects of interferon (IFN) $\alpha$ in IFN treated cells [24].

Purification of PV2A pro has remained an unmet challenge for picornavirologists. Early attempts at purification of PV2A pro illustrated that extracts of the protein from infected HeLa cells were very unstable [25]. Nevertheless Toyoda et al. [22], using a detergent based isolation procedure, succeeded in purifying 50 $\mu$ g of PV2A pro from PV-infected HeLa cells [22]. In the 1990s, using recombinant DNA technology, over-expression of recombinant PV2A pro in Escherichia coli was used to obtain sufficient protease to perform cleavage assays on eIF4G and the TATA-binding protein. In these experiments PV2A pro was either fused to maltose-binding protein (MBP) as a solubility tag [26] or purified under denaturing conditions followed by refolding [27]. However, the expression and purification of PV $2 \mathrm{~A}$ pro in sufficient quality and quantity for structural analysis has been unsuccessful.

In this manuscript, we report the cloning, expression, purification and computational analysis of recombinant PV2Apro. Following such structure-function investigations it is hoped that novel agents that inhibit PV through interference with proteolytic processing and thus virus replication might be screened and identified..

\section{Materials and Method's}

\subsection{Sequence retrieval}

The recombinant PV2Apro sequence was assembled by Eurofins from consensus sequences as reported in NCBI, GenBank (Accession numbers EU794958.1, EU794963.1, EU794961.1, EU794959.1, EU794957.1, EU794955.1, EU794953.1, EU794964.1, EU794962.1, EU794960.1, EU794954.1, and EU794956.1).

\subsection{Plasmid construction and site directed mutagenesis}

To facilitate cloning and expression of PV2Apro in the expression vector pET15b (Novagen) point mutations were incorporated into the PV2Apro sequence using the Quick-change site-directed mutagenesis kit (Stratagene) in combination with two oligonucleotides viz. sense, 5'TAATTCGGATGGGATTCGGACACCAAAAC-3' and antisense 5'GTTTTGGTGTCCGAATCCCATCCGAATTA-3'; mutagenised nucleotides are underlined and were confirmed by sequencing. PV2A pro protein was amplified from PV by PCR, gel purified, restricted with BamHI/XhoI and cloned into similarly restricted pET15b (+).

\subsection{Expression and solubilization analysis of $2 A^{\text {pro }}$}

The recombinant pET15b/2Apro plasmid was transformed into E. coli BL21 (DE3) pLysS cells Following overnight incubation on plates at $37^{\circ} \mathrm{C}$ individual colonies were inoculated into $10 \mathrm{ml} \mathrm{LB}$ broth containing $100 \mu \mathrm{g} / \mathrm{ml}$ ampicillin, $50 \mu \mathrm{g} / \mathrm{ml}$ kanamycin and $32 \mu \mathrm{g} / \mathrm{ml}$ chloramphenicol supplemented with $0.2 \%$ glucose and cultures which were grown at $37^{\circ} \mathrm{C}$ prior to inoculation as seed cultures into $5 \mathrm{~L}$ of Terrific broth containing the same antibiotics. Expression was induced by adding 
$1 \mathrm{mM}$ isopropyl-D-thiogalactoside (IPTG) when cultures approached an O.D.600 of 0.6. Control cultures contained uninduced recombinant plasmid. To confirm expression of recombinant protein 1 $\mathrm{ml}$ samples were collected at regular time intervals and analysed. Cultures were normally incubated for $4 \mathrm{~h}$ at $37^{\circ} \mathrm{C}$ and the cells pelleted by centrifugation $(38,500 \times \mathrm{g})$, and then lysed by sonication ( $5 x$ $15 \mathrm{sec})$ in lysis buffer $\left(40 \mathrm{mM} \mathrm{NaH}_{2} \mathrm{PO}_{4}, 300 \mathrm{mM} \mathrm{NaCl}, 500 \mathrm{mM}\right.$ imidazole, containing $0.2 \mathrm{mg}$ lysozyme and $10 \mu \mathrm{g} / \mathrm{ml}$ DNase1). Following centrifugation $(12,000 \times g$ for $15 \mathrm{~min}$ ) the pellet, containing inclusion bodies, was resuspended in lysis buffer containing $8 \mathrm{M}$ urea and incubated on ice for $2-3 \mathrm{~h}$ with stirring to denature proteins. All protein purification and storage was carried out at $4^{\circ} \mathrm{C}$. Subsequently proteins were renatured for 2 days with gradual dilutions of 6, 4, 2, and $0 \mathrm{M}$ urea containing lysis buffer prior to purification. At each step samples were collected and analyzed by SDS-PAGE (see below).

\subsection{Nickel nitrilotriacetic acid (Ni-NTA) purification and polyacrylamide gel electrophoresis}

Purification of the PV2A pro protein was achieved using affinity chromatography on a nickelnitrilotriacetic acid (Ni-NTA) gel matrix (Qiagen, Crawley, United Kingdom). The column was prepared by equilibrating $3 \mathrm{ml}$ of Ni-NTA resin in a column with 10 vols of lysis buffer comprising 3 vols of wash buffer $(50 \mathrm{mM}$ Tris- $\mathrm{HCl}, \mathrm{pH} 8.0,150 \mathrm{mM} \mathrm{NaCl})$ with increasing imidazole concentrations of 20,50, 100 and $200 \mathrm{mM}$. Bound protein was eluted with $20 \mathrm{mM} \mathrm{NaH}_{2} \mathrm{PO}_{4}, 200 \mathrm{mM}$ $\mathrm{NaCl}$ and $500 \mathrm{mM}$ imidazole buffer. The elution buffer was removed from the protein samples by dialysis and concentrations measured using a Nanodrop analyzer (Bio-Rad, Hercules, CA, USA) prior to assessing purity following SDS-PAGE and Coomassie blue staining.

All samples including time course samples, flow through, washings and eluates were analysed by SDS-PAGE on $14.5 \%$ gels as described by Laemmli [28]. Routinely 3X sample-reducing buffer was added to each sample, which was boiled for $5 \mathrm{~min}$ at $100^{\circ} \mathrm{C}$ before loading onto the gels. Proteins were fractionated by electrophoresis at $200 \mathrm{~V}$ for $c a .1 \mathrm{~h}$ or until the dye front had just exited the resolving gel. Gels were then stained with Coomassie blue.

\subsection{Matrix-assisted laser-desorption ionization-time-of-flight (MALDI-TOF) mass spectrometry}

Undigested peptides were analysed on a Voyager DE-STR MALDI-TOF mass spectrometer (Applied Biosystems, Paisley, UK). Samples were prepared for MALDI-TOF-MS using $50 \mu 1$ of $50 \%$ acetonitrile and $5.0 \%$ trifluoroacetic acid (TFA), dried and then purified on a ZipTip after adding 15 $\mu \mathrm{l}$ of $0.1 \%$ TFA. The peptides were eluted with $5 \mu \mathrm{l}$ of sinapinic acid (3,5-dimethoxy-4-hydroxy-transcinnamic acid), the matrix was dissolved in $50 \%$ acetonitrile, $0.1 \%$ TFA prior to analysis on a MALDI plate. Mass spectrometry analyses were performed in linear $\mathrm{m} / \mathrm{z}$ window on a MALDI-TOF-MS instrument (Amersham Biosciences). The $\mathrm{m} / \mathrm{z}$ spectra representing the monoisotopic masses of the undigested protein fragments were acquired in the MS and MS/MS modes. For manual matching, mass range for all spectra was set to 10,000-20,000 Da.

\subsection{Computational analysis}

The PV2A pro sequence was explored to identify the similar sequence by means of Basic Local Alignment Search Tool blast, beside database specification of non-redundant protein, accessible at the National Center for Biotechnology Information (NCBI) Web server [29]. All sequences were aligned using multiple alignment tool, Clustal Omega. Following automated programs were used to predict the secondary structures of the PV2Apro through their web site interfaces: PSIPRED [30-32], GOR4 [33], and SOPMA [34].

PDB file of 2A protease were generated by Swiss-Model server [35].To build the model of the 2A protein with more homology, high resolution structure of coxsakievirusB4 (1z8r) model in Swiss model server was selected as template. Validation of the models built was carried out using Ramachandran plot calculations [36].

\section{Results and Discussion}




\subsection{Site directed mutagenesis}

Site directed mutagenesis was used to add a methionine instead of a glycine residue as the translation start site for PV2Apro expression in E.coli after unsuccessful attempts to express the protein in the recombinant vector [37]. Following Dpn I digestion of PCR amplicons transformation of XL1Blue super competent cells was performed and recombinant clones were screened by sequencing to confirm successful mutagenesis.

\subsection{Expression and purification of $2 A$ protease}

The integrity of the pET15b expression vector used in these investigations was checked by sequencing prior to attempts to generate recombinant plasmids for over expression of the PV2A pro. Initially the PV2A pro was expressed in Rosetta-Gami 2 and BL21 cells but even the uninduced protein was toxic for both cell types and BL21 (DE3) pLysS were subsequently used in preference after optimization of expression conditions [38]. Non availability of a monoclonal antibody necessitated using an Anti His-tag antibody to purify for 2Apro.

Subsequently PV2Apro (estimated $M r 16.6 \mathrm{kDa}$ ) was over-expressed in E. coli following induction with $1 \mathrm{mM}$-IPTG at $37^{\circ} \mathrm{C}$ and Figure 1 shows a time course of expression of the protein with an apparent $\mathrm{Mr}$ of $17 \mathrm{kDa}$ after gel estimation.

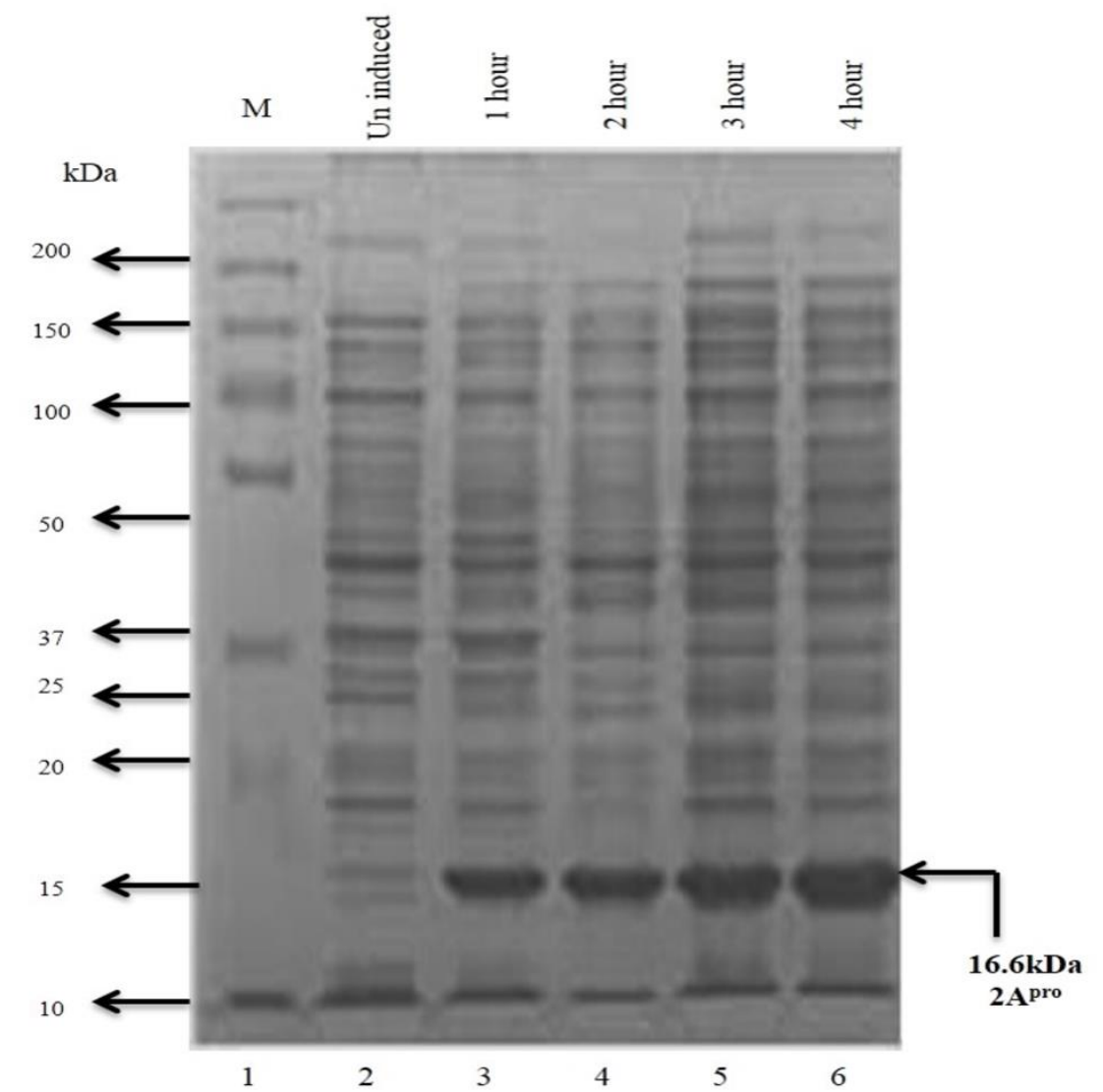

Figure 1. SDS-PAGE analysis depicting expression of poliovirus $2 \mathrm{~A}$ protease. Lane 1 shows the protein molecular weight marker, lane 2 represents the uninduced cell lysate of $E$. coli containing the recombinant plasmid for expression of PV2A pro, and lanes 3-6 show expression of PV2A pro following induction of the cultures with $1 \mathrm{mM}$ IPTG after $1 \mathrm{~h}, 2 \mathrm{~h}, 3 \mathrm{~h}$ and $4 \mathrm{~h}$ incubation respectively.

Following optimization of expression of the PV2A pro the insoluble protein fraction of lysed cells was solubilised in $8 \mathrm{M}$-urea and the solution dialysed sequentially removing the urea concentration to $0 \mathrm{M}$ to refold the protein prior to purification by $\mathrm{Ni}^{2+}$ affinity chromatography. However SDS PAGE analysis revealed that the purified protease had an $M r$ of $14.4 \mathrm{kDa}$ rather than the anticipated 
16.6 kDa (Figure 2). Following these observations the $M r$ of the protein was determined by mass spectrometry.
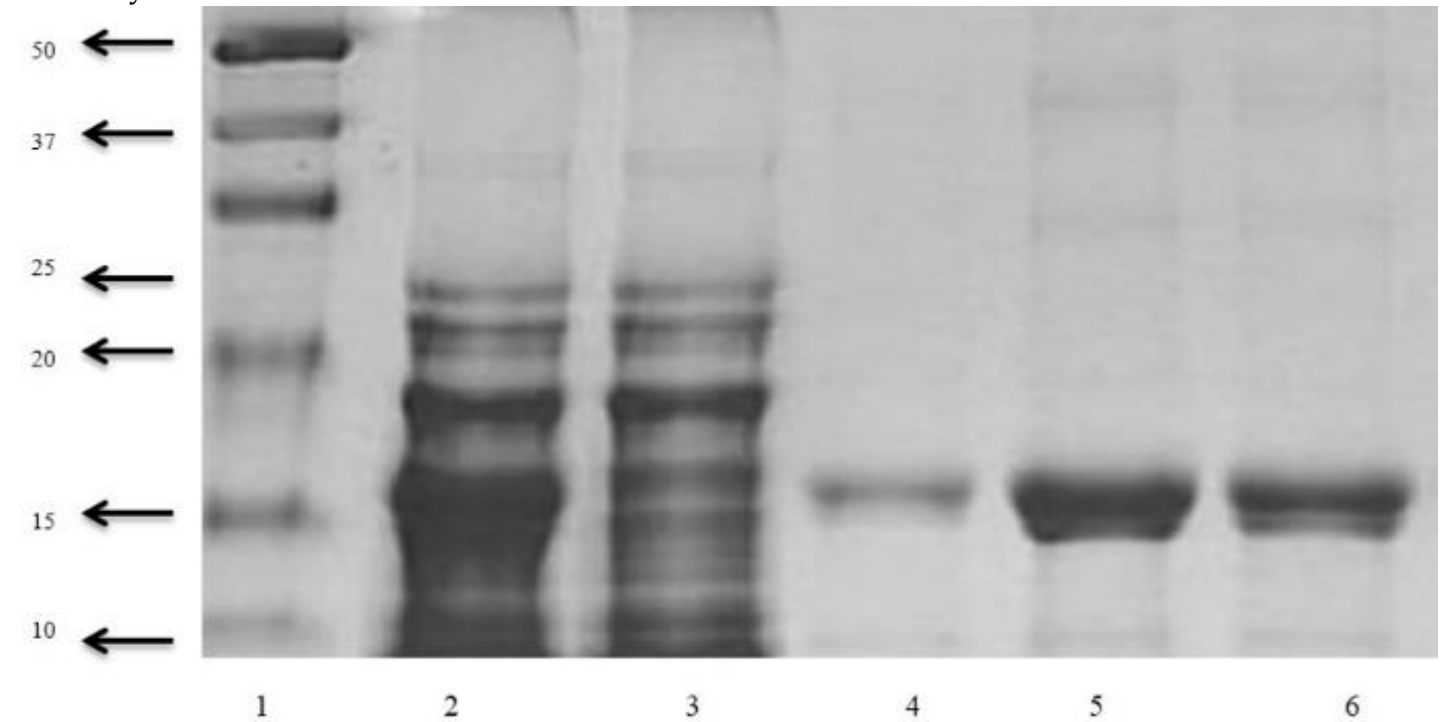

Figure 2. SDS PAGE analysis (14.5\% gel) of purified PV2A protease to determine the presence of protein in the eluted samples. Lane 1 is molecular size marker. Lane 2 contains a crude unfractionated lysate of induced cells; Lane 3 contains the column effluent (flow through); Lanes 4-6 contain fractions eluted with increasing concentrations of imidazole buffer respectively $100 \mathrm{mM}, 200 \mathrm{mM}$ and $500 \mathrm{mM}$.

Several attempts were made to express and purify 2A protease following the protocol used by Martinez-Abarca et al. However, sufficient amount of purified protein was not retrieved for crystallization assay [39]. Since the active form of protein was not obtained, therefore in silico approach was used to determine the protease binding sites against potential inhibitors (unpublished data). Because of our inability to crystallise PV2Apro, even though we were able to over express the protein in E. coli which could not be solubilized from inclusion bodies, we took advantage of its primary structural similarity to other viral proteases, specifically that from Coxsackievirus B4, and performed an in silico analysis of its structure.

To reduce the imidazole concentration in the purified denatured and refolded forms of $2 \mathrm{Apro}$, samples (200 and 500mM imidazole elutions) were pooled and concentrated using an ultrafiltration device with a $10 \mathrm{kDa} M r$ cut-off membrane (Amicon) following centrifugation at 50,228 $\times \mathrm{g}$. Several different buffers including HEPES, MES and $\mathrm{NaCl}+\mathrm{NaH}_{2} \mathrm{PO}_{4}$ were used unsuccessfully to solubilise the PV2Apro which appeared to be degraded in all cases. All attempts to concentrate and crystallise recombinant PV2Apro protein failed because of its localisation to the insoluble fraction of E.coli and its instability following solubilisation in urea and subsequent attempts at refolding after dialysis.

\subsection{Matrix-assisted laser desorption ionization time-of-flight (MALDI-TOF) mass spectrometry}

Peptide mass fingerprinting experiments were performed using MALDI-TOF MS to identify and characterise the content and integrity of the PV2A pro. A comparison of the MALDI-MS peptide maps obtained after digestion and extraction of pellet and supernatant samples confirmed insolubility of the 2Apro protein and that its $M r$. was $14.4 \mathrm{kDa}$, confirming the SDS-PAGE results.

\subsection{Computational analysis}

PV2Apro sequence was subjected to psi-BLAST that revealed 100 Blast hits on the query sequence against NCBI-PDB and the maximum percentage similarity with regard to sequence coverage and sequence identity was chosen for modeling. The template was selected considering the score, E-value and percentage identity. The sequence alignment was produced with Clustal Omega (40) multiple sequence alignment tool. The crystal structure of coxsakievirus B4 (Chain-A, PDB ID-1z8r) was found 
to be the best template. The psi-BLAST searching result showed that, the sequence identity between the target PV2A protease and the template $1 \mathrm{z} 8 \mathrm{r}$ is $56 \%$ with e-value 0 , query coverage $98 \%$, which allows for relatively straightforward sequence alignment (Figure 3).
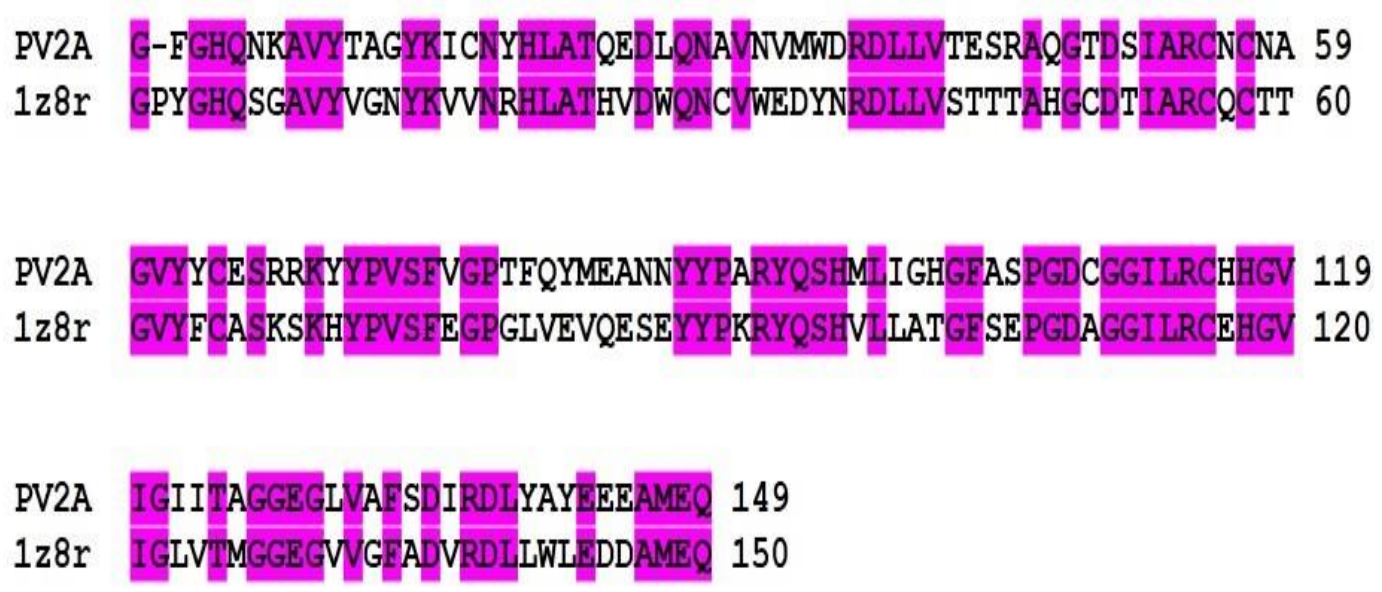

Figure 3. The alignment between standard PV2A (target) and 1z8r (template) obtained from ClustalW. Highlighted regions showed the conserved amino acids present in two sequences.

The three dimensional structure of recombinant PV2Apro was obtained via Swiss model. Template model was also generated through the same server (Figure 4).

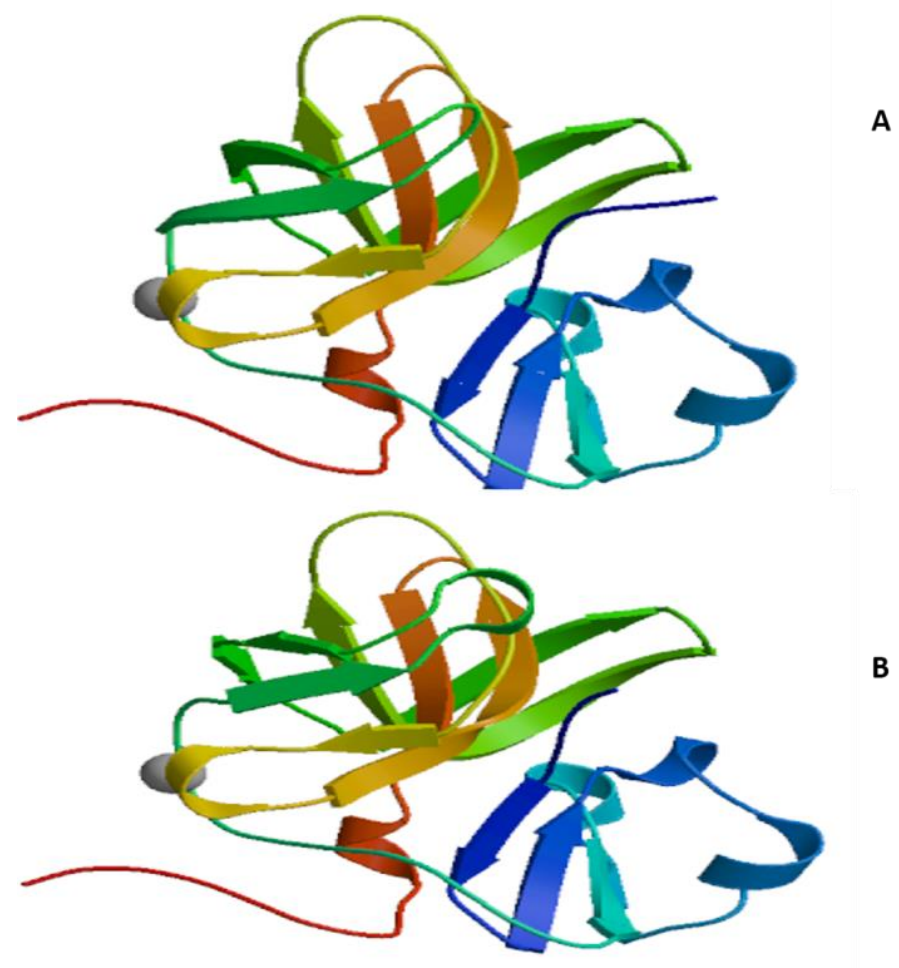

Figure 4. 3D model generation through Swiss model software. A. Template model 1z8r. B. Standard consensus model of poliovirus 2A protease.

Z-scores of final models were also determined that gives an indication of the overall quality of model. The $Z$ score values must be greater than -5 , and preferably $>-2$. In case of standard model $z-$ score was -3.27 (Table 1 ).

Table 1: QMEAN global scores for the final models (standard, 001 and 002, vaccine and template) obtained from Swiss modelling. Standard sample is considered best among all. 


\section{Z-score}

Scoring function term

1z8r

QMEAN6 score

$-4.18$

QMEAN4 score

All-atom pairwise energy

Ramachandran plot revealed, on average, more than $98 \%$ of the residues in the allowed region. The model has over $98 \%$ of residues in the favourable regions of the Ramachandran map and all the main-chain parameters, like peptide bond planarity, bad nonbonded interactions, C- $\alpha$ distortion, overall G-factor, bond length distribution and side-chain parameters, are in the normal range. Ramachandran plots of the model revealed that $90.5 \%$ of the residues lie in the most favoured position with $6.1 \%$ in additional allowed positions and $3.4 \%$ in disallowed regions. The small number of amino acid residues (Pro90, His101, Ser105, Ile112, and Cys115) in the disallowed region of the Ramachandran plot (Figure 5). 

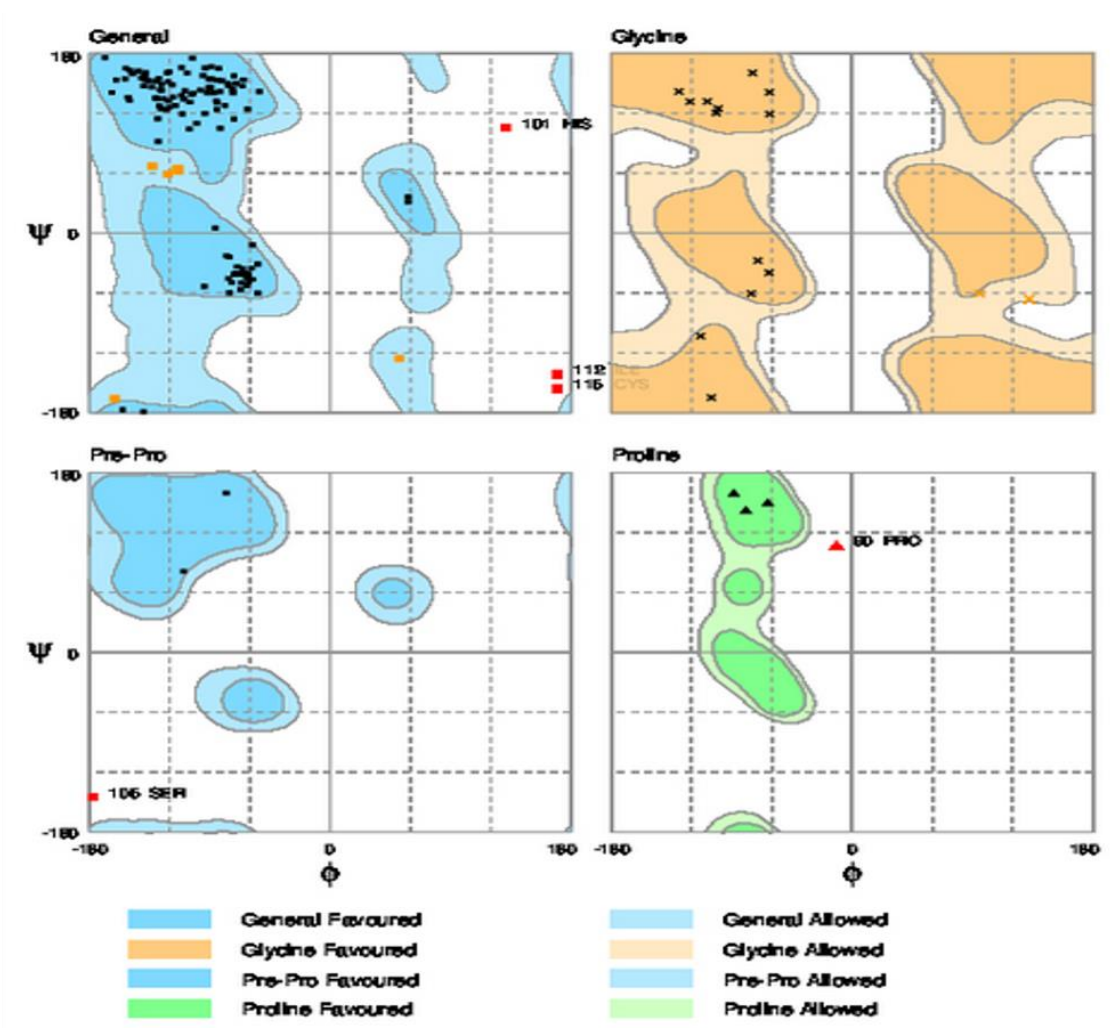

Number of residues in favored region ( 98.0\% expected) : 133 ( 90.5\%)

Number of residues in allowed region ( $2.0 \%$ expected) : 9 ( $6.1 \%)$

Number of residues in outlier region $\quad: 5(3.4 \%)$

Figure 5. Ramachandran plot analysis of recombinant PV2A pro model. Almost $98 \%$ of residues lie in favored region while about $2 \%$ in allowed region.

The above analysis of the predicted structure provides solid evidence that the predicted 3D structure of poliovirus2A protease is of good quality.

Secondary structure of poliovirus 2A protease was predicted using GOR4 software. Random coils are in abundance (55.03 \%) following extended strands $(24.16 \%)$ and alpha helices $(20.81 \%$; Figure 6). These results were further confirmed using SOPMA and PSIPRED softwares. 


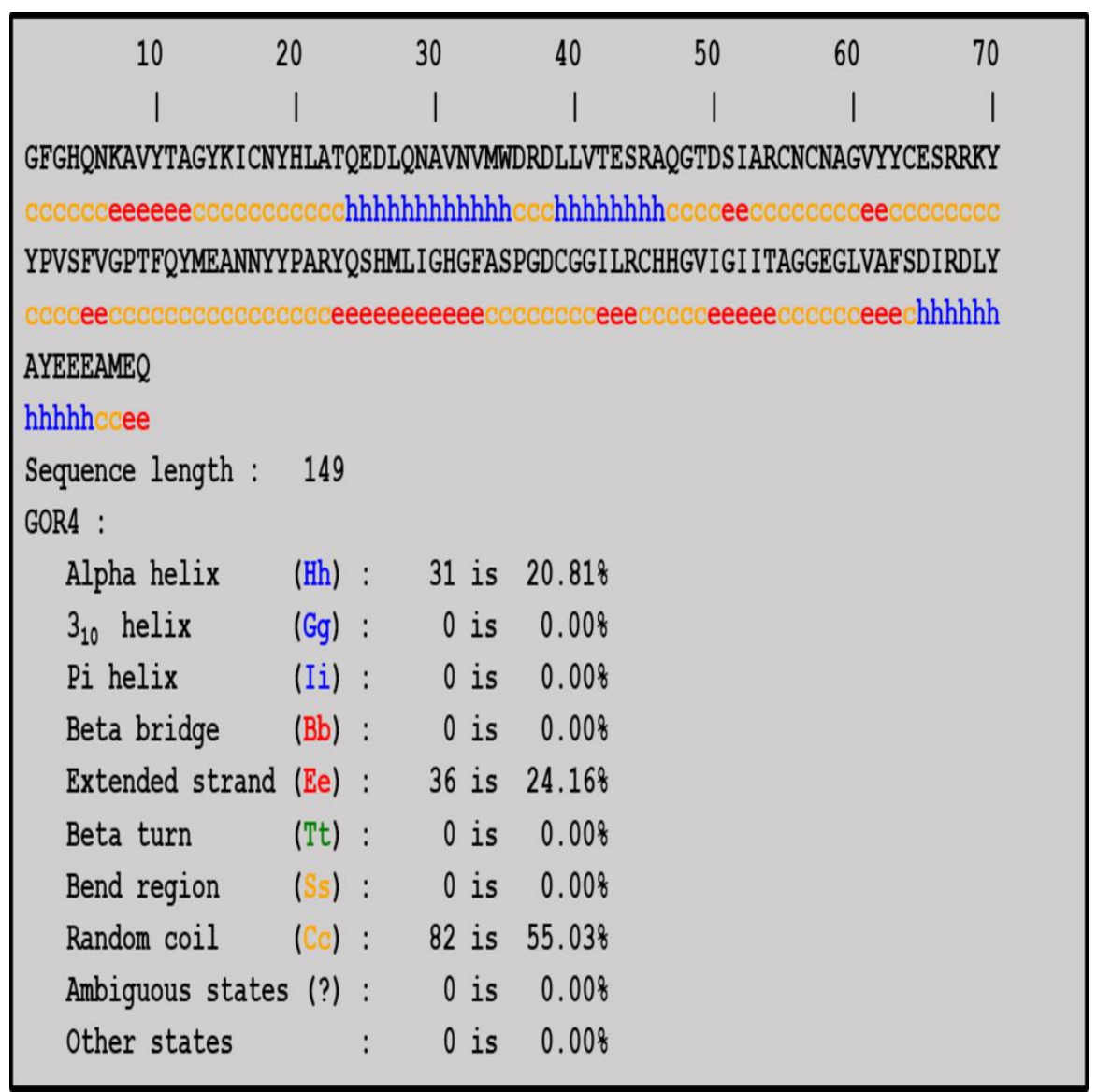

Figure 6. Secondary structure prediction of recombinant PV2Apro using GOR4 software. It shows that random coils are in abundance as compared to extended strands and alpha helices.

Because of the inherent instability of PV2A pro crystallization of the protein was not achieved in this investigation. We suspect that the convoluted structure of the PV2Apro, which contains many looped amino acid domains, was a major factor in its instability as noted previously when attempts were made to purify it directly from HeLa cells [25] and as a recombinant protein in E. coli [41]. Recent investigations have demonstrated that high-level expression and purification of picornavirus proteases in E. coli can be obtained with modifications [42] and we will investigate these procedures in further experiments.

In order to achieve the concentrated and pure form of $2 \mathrm{~A}$ protease for the sake of ion exchange and crystallization trials, several attempts were made to lower the imidazole concentration in the purified denatured and refolded forms of 2Apro. Different buffers (HEPES - NaOH, MES - NaOH, and $\mathrm{NaCl}+\mathrm{NaH}_{2} \mathrm{PO}_{4}$ ) were used to achieve the stability of the PV2Apro. Unfortunately after every buffer change concentration, protein seems to be unstable and degraded in the buffer. Due to extremely diverse structure and protein properties, it may not be possible to predict the "best" buffer for any given protein molecule [43]. Change in protein conformation or stability induced by a buffer can vary with buffer $\mathrm{pH}$, thereby resulting in the possibility that a buffer may become poorer (or better) at a different $\mathrm{pH}$ [44]. But attempts could be made to exploits the new range of buffers in order to achieve high stability of the protein.

Computational analysis of the protein proved it to be a better and suitable drug target against polio. Results are very much in accordance with the author's previous work using mutational samples of 2 A protease $[45,46]$. Use of computational approaches to identify more potential drug binding sites in the conserved regions will lead to better drug design methods in the years ahead. In the current analysis, for instance, protein binding sites that were identified at conserved regions are unique and can be directed for the attachment and inhibition of the target proteins. 


\section{Conclusions}

In this investigation several different E.coli strains were tested for their ability to express PV2Apro from a recombinant plasmid. E. coli BL21 (DE3) $p L y s S$ expressed the protein at $37^{\circ} \mathrm{C}$ following induction with IPTG but it was present in the insoluble fraction of the cells, toxic and all attempts to solubilize the protein were unsuccessful.

Using pure recombinant $2 \mathrm{~A}$ pro, it will be possible to hunt for $2 \mathrm{~A}$ pro inhibitors and select potent inhibitors of viral replication both in vitro and in vivo. This strategy mirrors a logical development for early stage drug discovery leading to successfully identify drug candidates.

Acknowledgments: The authors acknowledge the late Alastair Aitken for providing his guidance and instructions to conduct research at University of Edinburgh, UK. We are also thankful The School of Biological Sciences, University of Edinburgh for providing the protein expression, purification and MALDI-TOF facilities. We also acknowledge Malcolm Walkinshaw for reviewing the manuscript.

Author Contributions: Data curation, Muhammad Faraz Bhatti and Hussnain Janjua; Formal analysis, Amna Younus; Investigation, Amna Younus and Muhammad Faraz Bhatti; Methodology, Muhammad Faraz Bhatti, Nasar Virk and Muhammad Arshad; Project administration, Muhammad Faraz Bhatti, Nasar Virk, Muhammad Arshad, Hussnain Janjua and Robert Coutts; Supervision, Muhammad Faraz Bhatti; Writing - original draft, Amna Younus; Writing - review \& editing, Muhammad Faraz Bhatti, Nasar Virk, Muhammad Arshad, Hussnain Janjua and Robert Coutts.

Conflicts of Interest: We declare no conflict of interest.

\section{References}

1. Mueller, S.; Wimmer, E.; Cello, J. Poliovirus and poliomyelitis: A tale of guts, brains, and an accidental event. Virus Res. 2005, 111, 175-193.

2. Baltimore, D. Expression of animal virus genomes. Bacteriol. Rev. 1971, 35, 235-241.

3. Wimmer, E.; Hellen, C.U.; Cao, X. Genetics of poliovirus. Annu. Rev. Genet. 1993, 27, 353-436.

4. Holland, J.J.; Kiehn, E.D. Specific cleavage of viral proteins as steps in the synthesis and maturation of enteroviruses. Proc. Natl. Acad. Sci. USA. 1968, 60, 1015-1022.

5. Jacobson, M.F.; Baltimore, D. Polypeptide cleavages in the formation of poliovirus proteins. Proc. Natl. Acad. Sci. USA. 1968, 61, 77-84.

6. Sangar, D.V. The replication of picornaviruses. J. Gen. Virol. 1979, 26, 227-238.

7. Summers, D.F.; Maizel, J.V. Evidence for large precursor proteins in poliovirus synthesis. Proc. Natl. Acad. Sci. USA. 1968, 59, 966-971.

8. Teterina, N.L.; Levenson, E.A.; Ehrenfeld, E. Viable polioviruses that encode 2A proteins with fluorescent protein tags. J. Virol. 2010, 84, 1477-1488.

9. Ryan, M.D.; Luke, G.; Hughes, L.E.; Cowton, V.M.; ten Dam, E.; Li, X., Donnelly, M.L.L.; Menrotra, A.; Gani, D. The aphtho- and cardiovirus "Primary" 2A/2B polyprotein "Cleavage,". In Semler, B.L.;Wimmer, E. Eds.; Molecular biology of picornaviruses. ASM Press, Washington DC, 2002, pp, 213-223

10. Borman, A.M.; Kirchweger, R.; Ziegler, E.; Rhoads, R.E.; Skern, T.; Kean, K.M. elF4G and its proteolytic cleavage products: effect on initiation of protein synthesis from capped, uncapped, and IRES-containing mRNAs. RNA 1997, 3, 186-196.

11. Hambidge, S.J.; Sarnow, P. Translational enhancement of the poliovirus $5^{\prime}$ noncoding region mediated by virus-encoded polypeptide 2A. Proc. Natl. Acad. Sci. USA. 1992, 89, 10272-10276.

12. Liebig, H.D.; Ziegler, E.; Yan, R.; Hartmuth, K.; Klump, H.; Kowalski, H.; Blaas, D.; Sommergruber, W.; Frasel, L.; Lamphear, B.; Rhoads, R.; Kuechler, E.; Skern, T. Purification of two picornaviral 2A proteinases: interaction with eIF-4g and influence on in vitro translation. Biochemistry 1993, 32, 7581-7588.

13. Jurgens, C.K.; Barton, D.J.; Sharma, N.; Morasco, B.J.; Ogram, S.A.; Flanegan, J.B. 2Apro is a multifunctional protein that regulates the stability, translation and replication of poliovirus RNA. Virology 2006, 345, 346357.

14. Belov, G.A.; Lidsky, P.V.; Mikitas, O.V.; Egger, D.; Lukyanov, K.A.; Bienz, K.; Agol, V.I. Bidirectional increase in permeability of nuclear envelope upon poliovirus infection and accompanying alterations of nuclear pores. J. Virol. 2004, 78, 10166-10177. 
15. Graham, K.L.; Gustin, K.E.; Rivera, C.; Kuyumcu-Martinez, N.M.; Choe, S.S.; Lloyd, R.E.; Sarnow, P.; Utz, P.J. Proteolytic cleavage of the catalytic subunit of DNA-dependent protein kinase during poliovirus infection. J. Virol. 2004, 8, 6313-6321.

16. Park, N.; Katikaneni, P.; Skern, T.; Gustin, K.E. Differential targeting of nuclear pore complex proteins in poliovirus-infected cells. J. Virol. 2008, 82, 1647-1655.

17. Bernstein, H.D.; Sonenberg, N.; Baltimore, D. Poliovirus mutant that does not selectively inhibit host cell protein synthesis. Mol. Cell Biol. 1985, 5, 2913-2923.

18. Sun, X.H.; Baltimore, D. Human immunodeficiency virus activated expression of poliovirus protein $2 \mathrm{~A}$ inhibits mRNA translation. Proc. Natl. Acad. Sci. USA. 1989, 86, 2143-2146.

19. Krausslich, H.G.; Nicklin, M.J.; Toyoda, H.; Etchison, D.; Wimmer, E. Poliovirus proteinase 2A induces cleavage of eukaryotic initiation factor 4F polypeptide p220. J. Virol. 1987, 61, 2711-2718.

20. Sonenberg, N. Regulation of translation by poliovirus. Adv. Virus Res. 1987, 33, 175-204.

21. Goldstaub, D.; Alessandra, G.; Zippi, B.; Zehava, G.; Yaron, N.; Sylvie, L.; Nahum, S.; Chaim, K. Poliovirus 2A protease induces apoptotic cell death. Mol. Cell Biol. 2000, 20, 1271-1277.

22. Toyoda, H.; Nicklin, M.J.; Murray, M.G.; Anderson, C.W.; Dunn, J.J.; Studier, F.W.; Wimmer, E. A second virus-encoded proteinase involved in proteolytic processing of poliovirus polyprotein. Cell 1986, 45, 761770 .

23. Davies, M.V.; Pelletier, J.; Meerovitch, K.; Sonenberg, N.; Kaufman, R.J. The effect of poliovirus proteinase 2APro expression on cellular metabolism. J. Biol. Chem. 1991, 266, 14714-14721.

24. Morrison, J.M.; Racaniello, V.R. Proteinase 2A is essential for enterovirus replication in type I interferon treated cells. J. Virol. 2009, 83, 4412-4422.

25. Konig, H.; Rosenwirth, B. Purification and partial characterization of poliovirus protease 2A by means of a functional assay. J .Virol. 1988, 62, 1243-1250.

26. Novoa, I.; Martinez-Abarca, F.; Fortes, P.; Ortin, J.; Carrasco, L. Cleavage of p220 by purified poliovirus 2A (pro) in cell-free systems: effects on translation of capped and uncapped mRNAs. Biochemistry 1997, 36, 7802-7809.

27. Yalamanchili, P.; Banerjee, R.; Dasgupta, A. Poliovirus-encoded protease 2APro cleaves the TATA-binding protein but does not inhibit host cell RNA polymerase II transcription in vitro. J. Virol. 1997, 71, 6881-6886.

28. Laemmli, U.K. Cleavage of structural proteins during the assembly of the head of bacteriophage T4. Nature 1970, 227, 680-685.

29. Altschul, S.F.; Gish, W.; Miller, W.; Myers, E.W.; Lipman, D.J. Basic local alignment search tool. J. Mol. Biol, 1990, 215, 403-410.

30. Jones, D.T. Protein secondary structure prediction based on position-specific scoring matrices. J. Mol. Biol. 1999, 292, 195-202.

31. McGuffin, L.J.; Bryson, K.; Jones, D.T. The PSIPRED protein structure prediction server. Bioinformatics 2000, 16, 404-405.

32. Bryson, K.; McGuffin, L.J.; Marsden, R.L.; Ward. J.J.; Sodhi, J.S.; Jones, D.T. Protein structure prediction servers at University College London. Nucleic acids Res. 2005, 33, W36-W38.

33. Combet, C.; Blanchet, C.; Geourjon, C.; Deleage, G. NPS@: network protein sequence analysis. Trends Biochem. Sci. 2000, 25, 147-150.

34. Geourjon, C.; Deleage, G. SOPMA: significant improvements in protein secondary structure prediction by consensus prediction from multiple alignments. Computer applications in the biosciences: CABIOS. 1995, 11, 681-684.

35. Biasini, M.; Bienert, S.; Waterhouse, A.; Arnold, K.; Studer, G.; Schmidt, T.; Kiefer, F.; Cassarino, T.G.; Bertoni, M.; Bordoli, L.; Schwede, T. SWISS-MODEL: modelling protein tertiary and quaternary structure using evolutionary information. Nucleic acids research 2014, gku340.

36. Ramachandran, G.N.; Ramakrishnan, C.; Sasisekharan, V. Stereochemistry of polypeptide chain configurations. J. Mol. Biol. 1963, 7, 95-99.

37. Maniatis, T.; Fritsch, E.F.; Sambrook, J. Molecular Cloning." A Laboratory Manual. New York: Cold Spring Harbor Laboratory 1982.

38. Studier, F.W. Use of bacteriophage T7 lysozyme to improve an inducible T7 expression system. J. Mol. Biol. 1991, 219, 37-44.

39. Martinez-Abarca, F.; Alonso, M.A.; Carrasco, L. High level expression in Escherichia coli cells and purification of poliovirus protein 2Apro. J. Gen. Virol. 1993, 74, 2645-2652. 
40. McWilliam, H.; Li, W.; Uludag, M.; Squizzato, S.; Park, Y.M.; Buso, N.; Cowley, A.P.; Lopez, R. Analysis tool web services from the EMBL-EBI. Nucleic acids Res. 2013, 41, W597-W600.

41. Alvey, J.C.; Wyckofe, E.E.; Yu, S.F.; Lloyd, R.; Ehrenfeld, E. Cis- and trans-cleavage activities of poliovirus 2A protease expressed in Escherichia coli. J. Virol. 1991, 65, 6077-6083.

42. Nicklin, M.J.H.; Harris, K.S.; Pallai, P.V.; Wimmer, E. Poliovirus proteinase 3C: large-scale expression, purification, and specific cleavage activity on natural and synthetic substrates in vitro. J. Virol. 1988, 62, 4586-4593.

43. Ugwu, S.O.; Apte, S.P. The effect of buffers on protein conformational stability. Pharmaceutical Technology 2004, 28, 86-109.

44. Krajewska, B.; Zaborska, W. The effect of phosphate buffer in the range of $\mathrm{pH} 5.80-8.07$ on jack bean urease activity. J. Mol. Catal. B: Enzym, 1999, 6, 75-81.

45. Younus, A.; Bhatti, M.F.; Ahmad, T.; Virk, N.; Arshad, M.; Janjua, H.A. Protein structure modelling, ligand docking and active site analysis of mutated poliovirus 2A protease gene isolated from the blood of Pakistani polio infected patients. International Journal of Advances in Science Engineering and Technology 2016, 4.

46. Younus, A.; Bhatti, M.F. A comprehensive computational mutation structure-function approach for determining potential drug target sites in poliovirus 2A protease. Trop. J. Pharm. Res. 2017, 16, 2831-2841. 